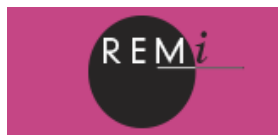

Revue européenne des migrations internationales

vol. 35 - $n^{\circ} 3$ et $4 \mid 2019$

Danses, musiques et (trans)nationalismes

\title{
Éditorial : Sciences en danger, revues en lutte
}

Editorial: Science in Peril, Journals in Struggle

Editorial: Ciencia en peligro, revistas en lucha

\section{Collectif des revues en lutte}

\section{(2) OpenEdition}

Journals

Édition électronique

URL : https://journals.openedition.org/remi/13192

DOI : $10.4000 /$ remi. 13192

ISSN : $1777-5418$

Éditeur

Université de Poitiers

Édition imprimée

Date de publication : 1 décembre 2019

Pagination : 7-13

ISBN : 979-10-90426-65-8

ISSN : 0765-0752

Référence électronique

Collectif des revues en lutte, «Éditorial : Sciences en danger, revues en lutte», Revue européenne des migrations internationales [En ligne], vol. 35 - n³ et 4 | 2019, mis en ligne le 01 janvier 2022, consulté le 15 avril 2022. URL : http://journals.openedition.org/remi/13192 ; DOI : https://doi.org/10.4000/remi. 13192 


\title{
Éditorial \\ Sciences en danger, revues en lutte
}

\section{Collectif des revues en lutte ${ }^{1}$}

\begin{abstract}
Le comité de rédaction de la Revue Européenne des Migrations Internationales, engagé dans le mouvement actuel des revues en lutte, a fait le choix de publier cet éditorial commun.
\end{abstract}

\begin{abstract}
Depuis le début de l'année 2020, plus d'une centaine de revues académiques, en grande majorité issues des sciences humaines et sociales françaises, se déclarent les unes " en lutte ", les autres " en grève ". Prenant part au mouvement social en cours, leurs comités de rédaction protestent à la fois contre le projet visant les retraites, contre la réforme de l'assurance chômage adoptée à l'automne 2019 et contre les propositions contenues dans les rapports pour la loi de programmation pluriannuelle de la recherche (LPPR) ${ }^{2}$. Par son ampleur et par sa forme - la grève et le vote de motions qui incitent les comités de rédaction à sortir de leur réserve habituelle -, cette mobilisation est historiquement inédite. La dynamique collective qu'elle suscite, par-delà les disciplines, les écoles et les conditions d'exercice de chacune des revues, témoigne du sentiment de révolte que provoquent ces réformes. Pour l'enseignement supérieur et la recherche, la réforme des retraites telle qu'envisagée actuellement par le gouvernement conduira à l'accroissement général des inégalités (entre hommes et femmes, entre titulaires et précaires, etc.) et à l'appauvrissement futur de toutes et tous, fonctionnaires, contractuel.le.s ou précaires. La réforme de l'assurance chômage augmentera, elle aussi, la vulnérabilité déjà difficilement supportable du très grand nombre des travailleur.euse-s précaires sur lesquel-le.s repose massivement la vie des universités et des laboratoires : ils et elles représentent d'ores et déjà plus d'un quart des effectifs d'enseignant·e-s, et encore bien davantage parmi les travailleurs et travailleuses administratif-ve.s et techniques. La LPPR, enfin, ne fera qu'aggraver le manque de moyens, de postes et de stabilité, et approfondir les inégalités qui minent l'enseignement supérieur et la recherche, et que deux décennies de "réformes " massivement contestées n'ont cessé d'amplifier.
\end{abstract}

1 À la date du 19 février 2020, un mois après le début du mouvement, 131 revues ont rejoint le mouvement des revues en lutte. Elles sont recensées sur le site universiteouverte.org

2 Les rapports sont signés par trois groupes de travail et consacrés au «Financement de la recherche ", à I' " Attractivité des emplois et carrières scientifiques " et à la " Recherche partenariale et innovation ". Ils sont disponibles sur le site du ministère de l'Enseignement supérieur, de la Recherche et de I'Innovation : https://www.enseignementsuprecherche.gouv.fr/cid145221/restitution-des-travaux-des-groupes-de-travail-pour-unprojet-de-loi-de-programmation-pluriannuelle-de-la-recherche.html 


\section{Une crise organisée du service public de la recherche et des universités}

Depuis bientôt trente ans, les gouvernements successifs contribuent à l'effritement de l'État social, au lent rognage de la fonction publique, à la dénonciation des "privilèges " gagnés dans les luttes sociales du XXe siècle, à I'affaiblissement des principes de redistribution destinés à réguler les disparités socioéconomiques et géographiques. Dans l'université et la recherche, la loi relative aux libertés et responsabilités des universités, dite "loi Pécresse " (LRU, 2007) a été la pierre angulaire d'un double mouvement, apparemment contradictoire : le désengagement budgétaire de l'État conformément à une logique néolibérale et le pilotage stratégique autoritaire de la recherche par ce même État. La logique de la loi tient à ce que l'autonomie (budgétaire) proclamée masque en réalité la dérégulation des statuts, la mise en concurrence de tou·te-s contre tou-te-s et la dépendance accrue de la recherche aux intérêts économiques et industriels, remettant finalement en cause l'autonomie véritable de la recherche. Cette politique menée avec opiniâtreté au mépris des mises en garde et des revendications de la communauté des chercheur-euse-s a multiplié les agences d'évaluation et de financement supposées indépendantes, prônant une culture de la "performance ", du " résultat " et de l' " excellence ", tout en réduisant les crédits propres des laboratoires au profit d'une distribution ciblée des moyens, largement définie par les aléas conjoncturels (sinon les modes), ainsi que par les hiérarchies et les situations préétablies. En privilégiant un financement par projets, elle a renforcé l'inégalité de dotations entre chercheur-euse-s et a conduit à un immense gaspillage d'énergie et d'argent public : combien d'heures perdues à évaluer ou à rédiger des projets pour obtenir d'hypothétiques financements, alors que ce temps aurait pu être consacré à la recherche ou à l'enseignement?

C'est peu dire, au reste, que les "gouvernants " nourrissent une obsession morbide pour les classements internationaux, dont la raison d'être est la promotion du modèle anglo-américain d'une université qui doit être gérée comme une entreprise, c'est-à-dire fonctionnant sur ses fonds propres (alimentés par des frais d'inscription appelés à augmenter), quitte à sacrifier le budget de fonctionnement et la qualité de I'encadrement. Depuis la LRU, la supposée mauvaise place des universités françaises dans ces classements est ainsi régulièrement invoquée pour tancer les chercheur.euse.s et poursuivre contre leur volonté la libéralisation de l'enseignement supérieur et de la recherche. Or ces injonctions se déploient dans un contexte de forte austérité budgétaire : rapporté au nombre d'étudiant·e·s, le budget de l'enseignement supérieur a ainsi chuté de plus de $10 \%$ depuis 2010 ; et malgré les promesses, répétées depuis vingt ans par les différents gouvernements, de porter à $1 \%$ du PIB l'effort budgétaire consacré à la recherche publique, celui-ci stagne toujours à 0,8\% (soit un manque de 6 milliards d'euros, une somme inférieure au crédit impôt recherche, cette niche fiscale concédée aux grands groupes industriels et de service). Dans ces conditions, les chercheur-euse-s et universitaires en France sont soumises à un régime qui mêle surtravail et dégradation des conditions de vie et de travail. Ils et elles sont de plus en plus précaires, et le restent de plus en plus longtemps, l'âge moyen du recrutement s'élevant à 35 ans. Les politiques d'austérité conduisent aussi à une réduction drastique de leurs revenus : alors que, 
en trente ans, les titulaires ont vu leur pouvoir d'achat chuter de $30 \%$, marquant ainsi un net décrochage avec le secteur privé, les travailleur-euse-s précaires connaissent une grande vulnérabilité, enchaînent contrats courts et périodes d'incertitude, et cumulent des vacations d'enseignements dont le montant se situe désormais en dessous du Smic horaire. Toutes et tous sont également de plus en plus évalué·e.s suivant des critères strictement comptables qui se limitent à dénombrer leurs publications, les contrats obtenus ou les brevets déposés, sans jamais interroger l'apport réel des connaissances produites. Ces différentes logiques font la part belle aux "entrepreneur.euse-s de carrière ", au détriment d'une recherche fondamentale, collective et véritablement indépendante.

Les mesures annoncées dans le cadre de la LPPR poursuivent avec obstination les transformations néolibérales engagées depuis le début des années 2000, dans le sillage de l'agenda de Lisbonne élaboré par le Conseil européen, et entendent les radicaliser. Empruntés au registre managérial, les mots d'ordre sont bien connus : compétitivité, financement par projet, concentration inégalitaire des moyens, austérité budgétaire, ce qui débouche sur un développement des emplois précaires et une mise en concurrence des individus, des laboratoires, des établissements, etc. Comme I'explique sans détour le PDG du CNRS Antoine Petit en novembre 2019, il s'agit d'engager une réforme " inégalitaire et darwinienne " : la concurrence généralisée et la concentration des ressources sur une minorité d'établissements et d'individus jugés plus " performants " selon des critères gestionnaires deviennent les principes cardinaux du gouvernement de I'enseignement supérieur et de la recherche, dans le cadre de "défis sociétaux" très perméables aux priorités de l'action gouvernementale. De nombreuses enquêtes démontrent pourtant les effets délétères de telles politiques sur l'originalité des savoirs produits et sur la qualité des formations dispensées aux jeunes générations : " effet Mathieu " - processus par lequel les plus favorisés augmentent leurs avantages - , standardisation de la recherche, bureaucratisation, affaiblissement de l'autonomie académique, appauvrissement de la diversité disciplinaire, etc.

Toute cette politique méprise ce que les chercheurs et chercheuses savent par expérience : dans toutes les disciplines, l'activité scientifique nécessite du temps et une disponibilité intellectuelle incompatible avec l'angoisse d'une précarité parfois radicale et avec la fragmentation croissante des tâches; elle s'exerce d'autant mieux que les équipes sont soudées alors que la compétition entre les pairs, désormais exacerbée, menace les collectifs ; et elle requiert une distance critique que la dépendance envers les hiérarchies administratives entrave. Ainsi, la communauté des chercheur-euse-s réclame avec force à la fois un engagement budgétaire à la hauteur des enjeux (en atteignant a minima l'objectif de $1 \%$ du PIB consacré à la recherche publique) et une distribution équitable des moyens à des personnels titulaires dont le statut de fonctionnaire demeure la condition de l'indépendance et de la sincérité des résultats.

\section{Revues scientifiques: une économie de la connaissance efficace}

Dans ce contexte, nos revues scientifiques occupent une place singulière et paradoxale. Lieux d'un intense travail collectif de production et supports 
efficaces de diffusion des savoirs, elles tendent à être instrumentalisées et mises au service de la vision néo-managériale dominante de la recherche. L'évaluation des chercheur.euse-s, des laboratoires et des universités repose en effet désormais en grande partie sur un décompte des articles publiés dans nos revues, selon des calculs bibliométriques dont la faiblesse et les effets pervers sur le plan scientifique ont été largement documentés ${ }^{3}$. Là n'est pas le moindre paradoxe des réformes en cours : alors qu'elles placent plus que jamais les revues au cœur de ce système de la recherche gouverné par "l'excellence " bibliométrique, elles conduisent non seulement à fragiliser leur fonctionnement, mais aussi à dénaturer le travail de production scientifique qui s'y déploie.

C'est pourquoi, en perturbant ou en interrompant notre activité, en refusant de nous tenir à distance de ce qui se joue dans la communauté scientifique comme dans le monde social, nous souhaitons mettre en avant aussi bien ce qui fait les revues que celles et ceux qui les font. Car notre travail collectif, intellectuel et éditorial, qui permet la production et le partage des savoirs, est directement menacé par les projets de loi actuels, qui fragilisent toujours plus le service public de l'enseignement supérieur et de la recherche.

L'existence de nos revues relève d'une économie de la connaissance fragile, mais néanmoins efficace. Ce sont aussi des scientifiques, dont une partie conséquente sont des agents publics, qui évaluent les textes, les discutent, les acceptent ou non en fonction d'expertises approfondies, font des suggestions à leurs auteurs et leurs autrices pour rendre ces textes plus pertinents, plus complets, plus exigeants dans leur démonstration, et qui, au terme de ce long processus de relectures, de discussions et de réécritures, de délibérations collectives et d'allers-retours avec les auteur-rice-s, publient et diffusent, sous forme d'articles scientifiques, les travaux qui sont à même de contribuer à la connaissance collective. Ces textes bénéficient, en outre, du travail minutieux de vérification formelle, de mise en forme et de mise en ligne, réalisé, quand ce n'est pas par les chercheur.euse.s, par des professionnel-le.s formé.e.s aux métiers de la documentation, de l'édition et/ou du numérique, dans le cadre de statuts variés, plus ou moins précaires - du fonctionnariat au CDD, en passant par le micro-entrepreneuriat. Enfin, ce sont surtout les bibliothèques universitaires, organismes publics, qui achètent les revues à l'unité ou en bouquets via des plateformes numériques. Cette offre en ligne, gratuite pour les étudiant·e-s, les enseignant.e.s et les chercheur.euse-s, et même tout un chacun quand il s'agit de revues en accès libre sur Internet, permet une large diffusion des dernières avancées scientifiques hors du champ universitaire : grâce au travail patient et collectif mené au sein de revues savantes, les enseignant.e.s ainsi que les journalistes, les associations, les élu·e·s, les citoyen-ne's bénéficient ainsi d'un apport substantiel et régulier de connaissances fiables et renouvelées.

Or, si cette économie de la connaissance assure l'enrichissement du savoir, elle rapporte toutefois peu en termes financiers. Elle est en effet adossée à une infrastructure invisible, celle du service public de la recherche.

C'est ce service public qui, idéalement, garantit des personnels formés, qualifiés et stables de secrétariat de rédaction.

3 Gingras Yves (2014) Les dérives de l'évaluation de la recherche. Du bon usage de la bibliométrie, Paris Raisons d'agir. 
C'est ce service public qui, idéalement, offre des réseaux ou des maisons d'édition, pour la numérisation, l'archivage ou la promotion des articles.

C'est ce service public qui, idéalement, permet l'existence de revues scientifiques numériques de qualité en accès ouvert et entièrement gratuites.

C'est ce service public, enfin, qui, malgré la lente dégradation des conditions de travail des statutaires et la précarisation des jeunes enseignant.e-s et chercheur-euse-s, continue de nous offrir le temps nécessaire pour siéger dans les comités de rédaction, pour concevoir les dossiers, lire, évaluer et discuter les articles proposés.

Pourtant, in fine, les quelques revenus produits par les revues ne servent à rémunérer ni les scientifiques qui les font vivre, ni les travailleurs et travailleuses qui les fabriquent. L'essentiel de ces revenus va en effet aux sociétés qui diffusent ces revues sur les plateformes de publication scientifique, dont beaucoup sont privées, au sein d'un secteur éditorial très fragile. À l'international et dans l'ensemble des disciplines scientifiques au-delà des sciences humaines et sociales, la situation est encore plus complexe. Ces sociétés y distribuent une part importante du travail d'édition : d'une part, elles économisent les tâches de relecture grâce au bénévolat des universitaires qu'elles sollicitent ; d'autre part, pour financer la pratique de l'accès ouvert, elles ont recours au modèle inversé de I'" auteur-payeur". Ainsi, la communauté scientifique et ses deniers publics payent plusieurs fois une activité dont les profits reviennent finalement à ces organisations commerciales prédatrices. Pour contrer cette marchandisation des savoirs, certaines plateformes et quelques revues ont proposé ces dernières années des dispositifs d'accès ouvert intégral, où l'auteur n'est pas payeur. Ceux-ci demandent à être renforcés et soutenus financièrement par les pouvoirs publics pour diffuser encore plus largement les savoirs scientifiques.

La LPPR, telle qu'annoncée, promet de saper les fondements de cette triple économie financière, scientifique et humaine, des revues. Elle frappe de plein fouet les personnels dits de soutien à la recherche, qui sont justement ceux qui permettent aux revues d'exister en tant qu'objets, en tant que produits manufacturés (même en ligne, même dans l'espace virtuel, un article est repris selon des normes typo-bibliographiques précises, mis en page et monté). Elle précarise ces personnels, substituant à l'emploi pérenne des contrats dits " de chantier ", qui obligeront nos revues à épuiser leurs forces pour solliciter, via de lourds dossiers de demande, le droit de bénéficier de quelques heures du contrat de travail d'une personne spécialisée dans l'édition. C'est là poursuivre une politique cynique de diminution drastique des emplois des personnels invisibles de la chaîne éditoriale (éditeur-rice-s, secrétaires de rédaction, les chargé·e·s d'édition, traducteur-rice·s, graphistes, développeur-euse-s, personnels des imprimeurs et des plateformes de publication numérique, etc.). En effet, parmi ces derniers, les rares personnes qui bénéficient d'un CDI ou du statut de fonctionnaire, sont généralement en sous-effectif et débordées par le flux constant de parution des revues, alors même que les tâches tendent à être " mutualisées " entre plusieurs publications, doublant voire triplant le travail de chaque poste. Quant à ceux et celles qui doivent jongler entre des CDD mal payés, ils et elles sont également contraint.e.s de travailler bien plus que les heures effectivement rémunérées, alternant périodes de chômage et embauches 
au sein d'équipes auxquelles, à peine formé·e·s, ils et elles n'ont guère le temps de s'intégrer. C'est ce que subissent les personnels d'OpenEdition, dont près de $60 \%$ sont contractuels, voire prestataires, alors que la plateforme est désormais devenue indispensable à la plupart de nos revues ${ }^{4}$. Les conséquences de ce système nous sont déjà connues et évoquent ce qui a été mis au jour dans le cas, notamment, de France-Télécom, de La Poste ou de l'hôpital public : surcharge de travail, détérioration des conditions de travail et des statuts générant souffrance, incertitude permanente, perte de sens et gaspillage des savoir-faire.

Enfin, privilégiant une recherche par projets assortie à des contrats limités dans le temps, diminuant drastiquement les recrutements de chercheureuse.s titulaires, la LPPR fragilise de façon dramatique les jeunes chercheur.euse-s en quête de poste, contraint-e.s de multiplier les CDD post-doctoraux pour vivre, ou de quitter la France pour aller là où on leur propose les postes qui manquent ici, voire de quitter la recherche pour un autre métier. Or, ce sont ces jeunes chercheur-euse-s qui contribuent massivement à la production d'articles scientifiques et au renouvellement des connaissances.

\section{Défendre l'autonomie de la recherche et de l'édition scientifique}

Comme on I'a vu, le travail de nos revues est un patient travail de discussion, et même, osons le mot malgré ses usages actuels, d'évaluation sur des critères partagés. La transparence et la pédagogie des processus éditoriaux, puis l'évaluation des articles, leur acceptation ou leur refus, ont des conséquences majeures sur les trajectoires des chercheur.euse-s et universitaires, notamment pour celles et ceux à la recherche de postes, et le rôle joué par les revues dans ce processus est indéniable. Mais, n'en déplaise à celles et ceux qui y verraient les outils par excellence de la sélection " inégalitaire et darwinienne ", nos revues ne sont pas des agences de notation destinées à établir le ranking des chercheur.euse-s, à classer les "talents " ou à mesurer les "performances". Car le processus d'évaluation est collégial, arbitré par la délibération dans des collectifs qui visent à produire la connaissance la plus précise, la plus robuste, la mieux démontrée. Si elles n'acceptent pas tous les articles qui leur sont soumis, nos revues ne sont pas des instances d'élimination qui mettraient en œuvre des critères $d^{\prime}$ " excellence " fixés par une bureaucratie quelconque : elles sont des lieux de réflexion et d'appréciation, mais aussi de communication avec les auteurs et autrices, pour définir ce qui, au regard de leurs projets intellectuels, " fait science ". Qu'elles soient généralistes, spécialisées ou interdisciplinaires, elles contribuent à informer la communauté scientifique, et bien au-delà, des recherches en cours, mais aussi à poser de nouvelles questions, à proposer des analyses ou des interprétations inédites, à lancer des controverses. Dans le vaste écosystème des revues académiques, chaque comité de rédaction travaille à élaborer une ligne éditoriale qui nourrit l'identité de la revue et ne saurait être réduite à une conception homogène de la scientificité. Ainsi, fondé sur des pratiques collectives et sur une conception coopérative et cumulative de la recherche scientifique, l'esprit qui anime nos revues est à l'opposé de la mise en concurrence et d'une illusoire évaluation individuelle des chercheur-euse-s.

4 https://academia.hypotheses.org/13060 
La coexistence de revues différentes est à ce titre indispensable : la pluralité et l'émulation sont les conditions du débat et de la confrontation, nécessaires aux progrès et à la validation des savoirs. La science s'élabore sur la contradiction, la multiplicité des approches et des écoles que, précisément, la concentration des moyens remet en cause. À l'opposé d'une conception managériale visant à faire des revues les centres de sélection et d'enregistrement d'une science uniformisée à l'échelle mondiale, c'est la capacité des différentes revues (nationales notamment) à défendre un point de vue scientifique particulier, une ligne spécifique, qui permet l'existence d'un espace international de points de vue, où la diversité des approches est une condition de la dynamique de la science. Ainsi, les revues scientifiques sont des instances de production et de diffusion d'une connaissance certifiée collectivement. Les articles et les dossiers qu'elles publient sont le fruit de travaux originaux : en sciences humaines et sociales, des mois de recherche dans des archives ou sur des terrains empiriques peuvent tenir en 50000 précieux signes. Ce processus d'évaluation, de délibération collective et d'échanges entre les comités de rédaction et les auteur-rice-s occupe de longs mois de travail, de sorte que rares sont les articles publiés dans leur version initiale. Auteur.rice-s, évaluateur.rice-s, membres du comité de rédaction, secrétaires de rédaction contribuent ainsi ensemble à la fabrication d'un savoir fiable et accessible. Lieux de transmission, de traduction et de production des idées et des recherches, espaces de rencontres et de débats, nos revues continuent de garantir un savoir scientifiquement solide et intellectuellement libre, à l'abri des intérêts privés. Elles contribuent à rendre la science meilleure.

Le monde de la recherche est déjà structuré par une très forte concurrence. Si l'on souhaite renforcer la qualité et la diversité de la production scientifique, ce n'est donc pas de darwinisme social dont nous avons besoin, mais plutôt d'espaces de travail stables, de " milieux " structurés sans lesquels les prises de risque, les coopérations et les débats indispensables à la production et à la consolidation de la connaissance ne peuvent se produire. En imposant des réformes structurelles permanentes, un pilotage vertical et par projets, l'accélération de procédures qui ne se conçoivent plus que dans le court terme, I'accroissement de la précarité des travailleur.euse-s et des collectifs de travail, le train de réformes dans lequel s'inscrit la LPPR ne fait que déstabiliser et appauvrir le fragile écosystème des revues.

Se mettre en grève, se mobiliser auprès des personnels en lutte, faire paraître un numéro blanc ou contribuer, par la publication de textes collectifs ou de récits anonymes, au mouvement social en cours : par ces actes inédits, et devant le constat de la dégradation du service public de la recherche, les revues expriment leur colère et leur inquiétude. Elles montrent d'un coup l'envers du décor et tout ce qui rend possible la production et la diffusion d'un savoir à la fois indépendant (notamment des mannes industrielles), fiable (car discuté par des scientifiques de haut niveau) et neuf (c'est ce savoir qui est à la base des futurs manuels universitaires, puis scolaires). Nos revues ne doivent leur existence qu'au service public de la recherche. Parce que le service public en général, et celui de la recherche en particulier, sont menacés, nous, collectif des revues en lutte, nous opposons aux projets de réforme en cours avec la plus grande fermeté. Nous refusons la casse des formes de collaboration et d'émulation solidaire qui font la force et l'honneur du modèle français de la recherche. 